\title{
Oil palm fruit bunch grading system using red, green and blue digital number
}

\begin{abstract}
This research deals with the ripeness grading of oil palm fruit bunches. The current practice in the oil palm mills is to grade the oil palm bunches manually using human graders. This method is subjective and subject to disputes. In this research, we developed an automated grading system for oil palm bunches using the RGB color model. This grading system was developed to distinguish between the three different categories of oil palm fruit bunches. The maturity or color ripening index was based on different color intensity. Our grading system employs a computer and camera to analyze and interpret images equivalent to the human eye and brain. The colors namely Red, Green and Blue (RGB) of the palm oil fruit bunch were investigated using this grading system. The computer program developed and used the mean color intensity to differentiate between the different color and ripeness of the fruits such as oil palm FFB. The program results showed that the ripeness of fruit bunch could be differentiated between different categories of fruit bunches based on RGB intensity.
\end{abstract}

Keyword: Grading system; Ripeness; color; Oil palm; Fruit bunch; Digital number 